\title{
Anterior cingulate cortex signals the requirement to break inertia when switching tasks: A study of the bivalency effect
}

\author{
Todd S. Woodward, ${ }^{\mathrm{a}, \mathrm{b}, *}$ Paul D. Metzak, ${ }^{\mathrm{a}, \mathrm{b}}$ Beat Meier, ${ }^{\mathrm{c}}$ and Clay B. Holroyd ${ }^{\mathrm{d}}$ \\ ${ }^{a}$ Department of Psychiatry, University of British Columbia, Vancouver, Canada \\ ${ }^{\mathrm{b}}$ Department of Research, Riverview Hospital, Coquitlam, Canada \\ ${ }^{\mathrm{c}}$ Department of Psychology, University of Bern, Switzerland \\ ${ }^{\mathrm{d}}$ Department of Psychology, University of Victoria, Victoria, Canada
}

Received 19 October 2007; revised 4 December 2007; accepted 16 December 2007

Available online 10 January 2008

\begin{abstract}
When switching tasks, if stimuli are presented that cue two of the tasks in the task set (i.e., bivalent stimuli), performance slowing is observed on all tasks, including those not cued by the bivalent stimulus. This slowing has been coined the bivalency effect, and may reflect adaptive tuning of the response style under conditions that appear to require adjustments in control over the course of action. Recent work on the function of the dorsal anterior cingulate ( $\mathrm{dACC}$ ) cortex has suggested that this neural region may be recruited under such conditions. In the current task switching study, we used tightly matched experimental and control conditions to isolate the bivalency effect. As predicted, dACC activation was associated with the bivalency effect, supporting an account stating that the role of the $\mathrm{dACC}$ is to signal a break in task inertia in order to adaptively tune the response style due to conditions that may require adjustments in control over the course of action. This result may extend the conflict monitoring account of dACC activation to situations where conflict occurred on past trials (i.e., conflict is not elicited by the current stimulus), and/or may support a more generalized account of $\mathrm{dACC}$ function involving monitoring internal states for conditions that may require adjustments in control over the course of action.

C) 2008 Elsevier Inc. All rights reserved.
\end{abstract}

Keywords: Anterior cingulate cortex; Stroop; Task switching; Bivalent stimuli

When switching between two or more tasks, if stimuli are encountered that cue at least two of the tasks being carried out (i.e., if a bivalent stimulus is encountered), slowing occurs on all tasks, regardless of their feature overlap with the bivalent stimulus. This

\footnotetext{
* Corresponding author. Room 2N9, Detweiller Pavillion, Department of Psychiatry, University of British Columbia, 2255 Wesbrook Mall, Vancouver, British Columbia, Canada V6T 1Z3. Fax: +1 6048227756.

E-mail address: toddswoodward@gmail.com (T.S. Woodward).

Available online on ScienceDirect (www.sciencedirect.com).
}

effect, coined the bivalency effect (Woodward et al., 2003), may reflect adaptive tuning of the response style under conditions that appear to require adjustments in control over the course of action. For example, if one's task is to sort apples arriving on a conveyor belt into bins holding red $(\mathrm{R})$, yellow $(\mathrm{Y})$, or green $(\mathrm{G})$ apples, the task can be carried out most quickly if (1) the sorting order always remains constant (RGYRGYRGYRGYR) and (2) the color of the arriving apples clearly matches the sorting order (i.e., also RGYRGYRGYRGYR). A number of manipulations can lead to performance slowing; however, in its most basic form the bivalency effect holds that even if sorting order remains constant, ambiguity of stimulus information on one task only will cause performance slowing on all tasks. For example, occasional unripe red apples that have clear green and red patches will slow down performance on all trials, even if the unripe red/green apples arrive only when red apples are expected. This slowing may prepare the cognitive system for the possible occurrence of more bivalent stimuli, a response to conditions that appear to require adjustments in control over the course of action (e.g., should this apple be sorted into the red or green bin). Experimentation in our laboratory (Woodward et al., submitted for publication) has shown that bivalency is required for this effect to emerge because stimuli that are surprising but not bivalent (e.g., oddly shaped apples) do not cause generalized performance slowing, and awareness that bivalent stimuli will arrive does not extinguish the effect.

The neuroimaging literature suggests that activation of the dorsal anterior cingulate cortex (dACC) should be associated with the bivalency effect. Proposed functions of the dACC include modulation of conflict between neuronal processing streams (Parris et al., 2007; Peterson et al., 1999), selection between competing mental processes (Holroyd and Coles, 2002), generic conflict detection (Botvinick et al., 1999; Cohen et al., 2000), voluntary selection of task sets (Forstmann et al., 2006), overcoming inertia (Paus, 2001), task-set reconfiguration (Crone et al., 2006; Woodward et al., 2006b), and allocation of arousal's energy to the required response set (Stuss, 2006). At their core, all of these interpretations involve breaking inertia to prepare a change in 
response style. Syntheses of human imaging studies, mathematical modeling and animal work have concluded that the ACC may signal that an adjustment in control or course of action is required (Behrens et al., 2007; Gehring and Taylor, 2004; Hayden and Platt, 2006; Luks et al., 2002; Ridderinkhof et al., 2004; Rushworth et al., 2004; Volz et al., 2003; Williams et al., 2004). Due to its role in breaking the inertia set into motion by carrying out a series of tasks, the dACC would be expected to be activated when the bivalency effect is observed.

In the current task switching study, we used tightly matched experimental and control conditions to isolate the bivalency effect. In both the control and experimental conditions, participants switched between three tasks, but in the experimental condition bivalent stimuli were encountered occasionally on one of the tasks, thereby invoking slowing on all three tasks (i.e., the bivalency effect). Thus, participants switched between identical tasks, using identical stimuli, for Blocks 1 and 3 (control blocks) and Block 2 (experimental block). The need to break inertia was invoked by inserting occasional bivalent stimuli in a series of univalent stimuli (bivalent and univalent refer to stimuli that cue two and one of the tasks in the task set, respectively) in Block 2. An event-related design allowed us to exclude these bivalent stimuli from analysis of the activations, and compare activation from univalent stimuli in Blocks 1 and 3 to those from univalent stimuli in Block 2, thereby equating the influence of Stroop-like conflict between the experimental and control conditions. We expected to observe an increase in the dACC activation associated with the bivalency effect on all tasks, despite excluding the bivalent trials that invoke Stroop-like conflict.

\section{Method}

Participants

The participants were 29 healthy, right handed, English speaking volunteers $(13$ men and 16 women, mean age $=24.93, \mathrm{SD}=$ 5.13 years) with normal or corrected to normal vision. Participants were recruited via advertisements and word-of-mouth from the community of Vancouver, British Columbia, and participated in exchange for $\$ 10$ per hour and a copy of their structural brain images. All participants were screened for MRI compatibility, and gave informed written consent prior to participation. All experimental procedures were approved by the University of British Columbia clinical research ethics board.

\section{Materials}

Stimuli were displayed against a black background. A set of univalent stimuli (i.e., stimuli that cue only one task in the task set) were prepared. For the color decision task, the stimuli were one of four shapes (circle, triangle, square, or pentagon), displayed in either blue or red. For the parity decision task, the stimuli were the numerals 1 through 8, displayed in white, in 60-point Times New Roman font. For the case decision task, the stimuli were the letters $\mathrm{a}, \mathrm{b}, \mathrm{d}, \mathrm{e}$, displayed in white, in either upper- or lower-case, in 60point Times New Roman font. The bivalent stimuli were prepared (i.e., stimuli that cue two of the tasks in the task set) for the case decision task only as follows: the letters: $a, b, d$, e, displayed in either blue or red, in either upper- or lower-case, in 60-point Times

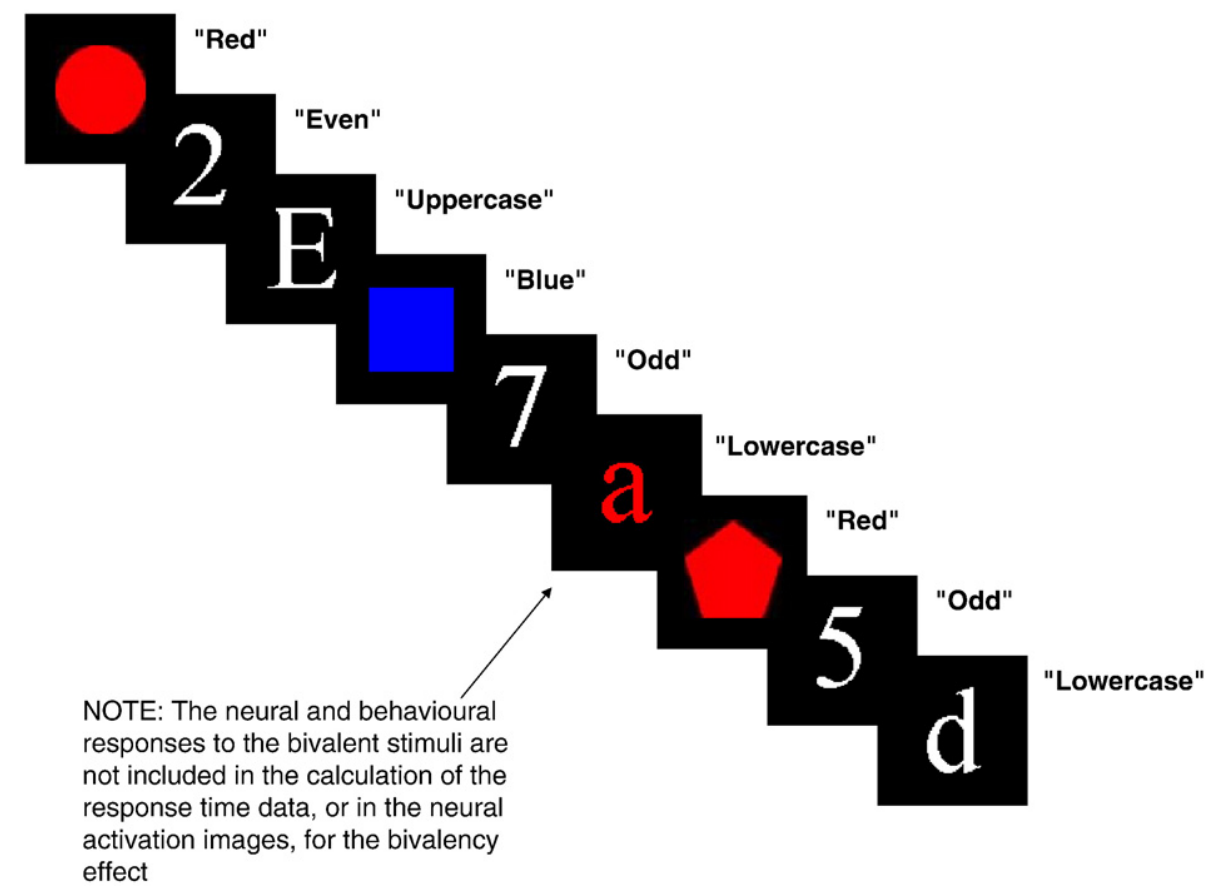

Fig. 1. Example of stimuli for the scanned task switching paradigm. The experimental run lasted $864 \mathrm{~s}$ and was divided into three blocks. Each block consisted of 40 triplets of the same three tasks performed in the same order. Each stimulus was displayed for $1500 \mathrm{~ms}$ and was followed by a blank screen for $500 \mathrm{~ms}$. During the 1st and 3rd blocks of the experimental run, only univalent stimuli were displayed. During the 2nd block of the experimental run, 20\% (8/40) of the case decision stimuli were bivalent, and these bivalent stimuli were pseudo-randomly distributed throughout the block. Note that the responses to the bivalent stimuli are excluded from the computation of the response time data, or neural activation images, for the bivalency effect. 
New Roman font. All stimuli were matched on pixel size. Please see Fig. 1 for an example of the stimuli and task.

\section{Procedure}

\section{Practice}

Participants were initially screened for handedness using the Annett handedness scale (Annett, 1970), and color vision using a portable eye chart. All participants were right handed, had intact color vision, and visual acuity that was $20 / 20$ or corrected to $20 / 20$. Participants were instructed that they would be performing two practice versions of the experiment prior to entering the scanner. They were told that all versions of the experiment would require them to perform 3 tasks in alternation (referred to below as a triplet): judging if a shape was blue or red (color task), judging if a number was odd or even (parity task), and judging if a letter was uppercase or lowercase (case task). Thus, each triplet consisted of three tasks: a color judgment, a parity judgment, and a case judgment, in that order. During practice, only univalent stimuli were presented. Participants were randomly assigned to either the right hand response or left hand response condition, in order to negate activations solely attributable to the act of responding. In both conditions, responses on the practice versions were made by pressing the left and right arrows on the keyboard. The "blue", "odd", and "lower" responses were assigned to the left arrow key, and the "red", "even", and "upper" responses were assigned to the right arrow key.

For both of the practice versions, the stimuli remained on the screen for $1500 \mathrm{~ms}$. The first practice version of the experiment was used to familiarize the participants with the experimental tasks. After each stimulus was presented, the program provided feedback indicating whether the last response was correct, incorrect, or too slow. The second practice version was designed to acclimatize the participants to the pacing of the experiment as they would experience it in the scanner; thus, a $500 \mathrm{~ms}$ ISI was implemented. There was no immediate feedback following each task; however, the participant's percentage correct was displayed on the screen four times throughout the course of the experiment. In both practice versions, the experiment ended when the participants achieved a score of $90 \%$ correct or greater over 10 triplets; however, participants were required to complete a minimum number of triplets before this condition came into effect: in the first practice experiment, the participants were required to complete at least 20 triplets, and in the second practice experiment, the participants were required to complete at least 40 triplets. No participant needed more training than this to achieve the required response accuracy. Upon completion of the second practice version, participants were taken to the MRI suite.

\section{Scanning trials}

For the scanning trials, the stimuli were projected onto a screen fastened at the entrance of the magnet bore using Presentation software (version 9.70, www.neurobs.com). Participants viewed the screen using a mirror mounted onto the head coil. The experimental instructions were reiterated and the participants were told to indicate their response using a LUMItouch fiber-optic response device (Lightwave Medical, Vancouver, British Columbia, Canada) with the same hand and finger-response assignments that they had used during the practice phase. Participants were also told that "Relax" trials would appear on the screen at various points throughout the experiment, and they were instructed to relax and avoid thinking about the experiment when this occurred.
The experiment consisted of 3 blocks with 40 triplets per block. As in the practice session, each triplet consisted of a color judgment task, a parity judgment task, and a case judgment task, in that order (see Fig. 1). Each stimulus was presented for $1500 \mathrm{~ms}$ and was followed by a blank screen for $500 \mathrm{~ms}$. During the first and third blocks of the experiment, only univalent stimuli were presented, just as had been the case in the practice trials. In order to produce the bivalency effect, in accordance with past work (Woodward et al., submitted for publication, 2003), during the second block, 20\% (8/40) of the "case task" stimuli were bivalent (i.e., colored), and were pseudo-randomly distributed throughout the block, occurring on triplet $1,6,12,16,20,27,32$, and 37. Presentation of the bivalent stimuli on the case task did not require any additional instructions because participants were not required to process the irrelevant color dimension. The entire experimental run lasted $864 \mathrm{~s}$. "Relax" trials appeared for $40 \mathrm{~s}$ at the beginning and the end of the functional run. A "Relax" trial was also presented for $10 \mathrm{~s}$ following the 20th, 40th, 60th, 80th, and 100th triplets, and 120 triplets were presented in total, in one run.

\section{Image and image processing}

Imaging was performed at the University of British Columbia's MRI Research Centre on a Phillips Achieva 3.0 Tesla MRI scanner with Quasar Dual Gradients (maximum gradient amplitude $80 \mathrm{mT} / \mathrm{m}$ and a maximum slew rate of $200 \mathrm{mT} / \mathrm{m} / \mathrm{s}$ ). The participant's head was firmly secured using a custom head holder. Functional images volumes were collected using a T2*-weighted gradient echo spin pulse sequence $\left(\mathrm{TR} / \mathrm{TE}=2000 / 30 \mathrm{~ms}\right.$, flip angle $90^{\circ}, 36$ slices, $3 \mathrm{~mm}$ thick, $1 \mathrm{~mm}$ gap, sense factor $2,80 \times 80$ matrix reconstructed at 128 , FOV $240.0 \mathrm{~mm}$, measured voxel is $3 \mathrm{~mm} \times 3 \mathrm{~mm} \times 3 \mathrm{~mm}$, actual band width $=53.4 \mathrm{~Hz}$ per pixel) effectively covering the whole brain (145 $\mathrm{mm}$ axial extent). A total of 432 images of the entire brain were collected over a period of $864 \mathrm{~s}$.

Functional images were reconstructed offline, and the scan series was realigned and normalized using the method implemented in Statistical Parametric Mapping 2 (SPM2; http://www.fil.ion.ucl. ac.uk/spm). Translation and rotation corrections did not exceed $3 \mathrm{~mm}$ or $3^{\circ}$ for any of the participants. Parameters for spatial normalization into the modified Talaraich space used in SPM2 were determined using mean functional images constructed from the realigned images of each participant and scan series. Voxels were normalized to $2 \mathrm{~mm} \times 2 \mathrm{~mm} \times 2 \mathrm{~mm}$. The normalized functional

Table 1

Mean (SE) reaction times in milliseconds for correct trials only

\begin{tabular}{llll}
\hline Response times & & & \\
\hline Block & Color & Parity & Case \\
\hline Univalent stimuli & & & \\
1 & $652(15)$ & $684(16)$ & $648(15)$ \\
2 & $719(20)$ & $708(17)$ & $654(17)$ \\
3 & $668(18)$ & $663(14)$ & $625(13)$ \\
Bivalent stimuli & & & \\
2 & & & $820(28)$ \\
\hline
\end{tabular}

Note. The univalent stimulus means are based on the correct trials in each block (maximum of 40 in Blocks 1 and 3, maximum of 32 in Block 2). The bivalent stimulus means are based on the correct trials in Block 2 only, which is the only block in which bivalent stimuli were presented (maximum of 8). The listed values here are the mean response times in milliseconds (standard errors in parentheses), averaged over task order. 
Table 2

Significant clusters obtained from analyzing the contrast between correct univalent stimuli in Block 2 vs. correct univalent stimuli averaged over Block 1 and Block 3 (i.e., the bivalency effect)

\begin{tabular}{|c|c|c|c|c|c|c|c|}
\hline \multicolumn{3}{|l|}{ Clusters } & \multicolumn{5}{|c|}{ Peak activations } \\
\hline Cortical regions & Size & $\begin{array}{l}\text { Overlapping } \\
\text { BAs }\end{array}$ & $X$ & $Y$ & $Z$ & $t$ & $\mathrm{BA}$ \\
\hline Cluster 1 & 699 & $6,8,9,10,32,42$ & 4 & 40 & 34 & 6.01 & 9 \\
\hline $\begin{array}{l}\text { Anterior cingulate } \\
\text { gyrus }\end{array}$ & 27 & 32,42 & & & & & \\
\hline $\begin{array}{l}\text { Medial frontal } \\
\text { gyrus }\end{array}$ & 437 & $6,8,9,10$ & & & & & \\
\hline $\begin{array}{l}\text { Superior frontal } \\
\text { gyrus }\end{array}$ & 182 & 8,9 & & & & & \\
\hline Cluster 2 & 118 & $7,19,39$ & -32 & -72 & 44 & 4.88 & 7 \\
\hline Precuneus & 53 & 7,19 & & & & & \\
\hline $\begin{array}{l}\text { Inferior parietal } \\
\text { lobule }\end{array}$ & 3 & $7,19,39$ & & & & & \\
\hline $\begin{array}{l}\text { Superior parietal } \\
\text { lobule }\end{array}$ & 53 & 7 & & & & & \\
\hline
\end{tabular}

Note. Activations are listed by cluster (left column) and peak activations (right column). A random effects analysis was carried out, with cutoffs of $p<0.001$ uncorrected at the voxel level, and $p<.05$ corrected at the cluster level. Talairach region voxel counts do not equal cluster total voxel counts due to interhemispheric or non-cortical locations. BA=Brodmann's Area. $t=t$ value for peak activation.

images were smoothed with a $6 \mathrm{~mm}$ full width at half maximum Gaussian filter.

\section{Results}

\section{Behavioral}

For the analysis of the behavioral data (and the analysis of the imaging data), all trials on which an error was committed were excluded. For each Task Type and Block, Table 1 shows the mean reaction times and their respective standard errors. The bivalency effect was of primary interest for this study; therefore, the RT measure for the bivalency effect was computed for each participant as follows: the mean of Block 1 and 3 RTs, subtracted from that computed on the univalent stimuli presented during Block 2, resulting in a computation of the bivalency effect for each task.

The bivalency effect RT difference scores (i.e., the mean of Blocks 1 and 3 subtracted from the mean of Block 2, a quadratic contrast) were analyzed by way of a repeated-measures analysis of variance (ANOVA), with one factor, Task, with three levels (color, parity, and case). This ANOVA revealed a significant main effect of Task, $F(2,56)=10.27, p<0.001, \eta^{2}=.27 . t$-tests were assessed at .05 one-tailed, under the prediction of slower RT to univalent stimuli for Block 2 as is required for the bivalency effect, and this resulted in a significant bivalency effect for all tasks; however, the magnitude of this effect was in decreasing order for color, parity and case, $t(28)=$ $6.09, p<.001, \eta^{2}=.57 ; t(28)=3.71, p<.001, \eta^{2}=.33 ; t(28)=1.68$, $p=.05, \eta^{2}=.09$, respectively, explaining the significant Task effect. A parallel analysis on errors reported no Task effect for errors, $F(2,56)=$ $2.08, p=0.13, \eta^{2}=.07$. However, slightly more errors were committed in Block 2 compared to the average of Blocks 1 and $3, t(28)=2.54$, $p<.05, \eta^{2}=.19$, with the mean number of errors (averaged over tasks) being $.943(\mathrm{SE}=.171), 1.540(\mathrm{SE}=.222)$, and $1.322(\mathrm{SE}=.270)$ for Blocks 1, 2, and 3, respectively, out of 40 trials. Despite the very low error rate, as mentioned above, in order to avoid the potential confound of error commission with the bivalency effect, all trials on which an error was made were excluded from the analysis of the RT and fMRI data.

In order to be consistent with work comparing RT on bivalent stimuli with that on univalent stimuli (e.g., computation of the "Stroop" effect), we computed the RT difference on the eight bivalent stimuli in Block 2, and subtracted that from univalent case trials in the same position in Blocks 1 and 3. More specifically, the bivalent case stimuli RTs were computed for triplet 1, 6, 12,16, 20, 27, 32, and 37 in block two $(M=820.28, \mathrm{SD}=27.89)$, and so were compared to the average of the univalent case response times in triplet $1,6,12,16,20,27,32$, and 37 in Blocks 1 and $3(M=681.81, \mathrm{SE}=18.38)$. This resulted in a significant "Stroop" effect of $138.47 \mathrm{~ms}, t(28)=5.13, p<.01, \eta^{2}=.48$.

\section{Neuroimaging}

For analysis of the brain images, statistical parametric mapping (SPM2) was used. The event-related responses to all events were modeled using a synthetic hemodynamic response function composed of two gamma functions (Josephs et al., 1997). For each of the scan series, the model of the composite hemodynamic response for the entire run comprised a sequence of appropriately placed synthetic responses to ten different event types: (1) correct color trials from Block 1, (2) correct color trials from Block 2, (3) correct color trials from Block 3, (4) correct parity trials from Block 1, (5) correct parity trials from Block 2, (6) correct parity trials from Block 3, (7) correct case trials from Block 1, (8) correct case trials (univalent only) from Block 2, (9) correct case trials

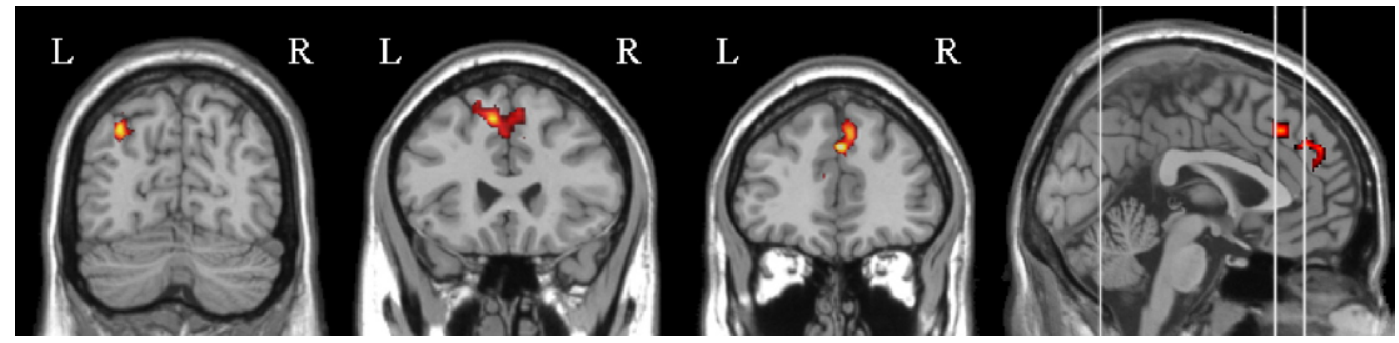

Fig. 2. Image of activations associated with the bivalency effect. A random effects analysis with cutoffs of $p<0.001$ uncorrected at the voxel level, and $p<.05$ corrected at the cluster level, was used. The correct responses to univalent color, number, and case stimuli in Block 2 were contrasted with the correct responses to univalent color, number, and case stimuli averaged over Block 1 and Block 3. $t$ values assigned colors in the figure ranged from 3.41 to 6.01. Anterior cingulate activation was found to underlie the bivalency effect (i.e., slowing on RT in Block 2). $Y$ coordinates for the coronal images are $y=-70, y=26, y=40$, and for the sagittal image $x=0$. The white lines on the sagittal image reference the slices displayed to the left in the coronal plane. 
from Block 3, and (10) correct case trials (bivalent only) from Block 2.

As was mentioned above, based on previous work (Woodward et al., submitted for publication, 2003), the quadratic effect (i.e., the contrast of Block 2 with the mean of Blocks 1 and 3) was of primary interest for the question of whether or not dACC activation is associated with the bivalency effect. We specified a quadratic contrast for the comparison of all the univalent stimuli presented during Block 2 vs. the average of the univalent stimuli presented in Blocks 1 and 3 using the method implemented in SPM2. All clusters listed are in MNI coordinates. Using a random effects analysis on bivalency contrasts, an uncorrected $p$-value of 0.001 at the voxel level, and .05 corrected at the cluster level, two significant clusters of activation emerged. The first cluster had a peak activation at $X Y Z=4$, 40, 34 (BA 9) and an extent of 699 voxels. The activation for this cluster extended bilaterally into the anterior cingulum and cingulum gyri (BA 32 and 42), medial frontal gyri (BA 6, 8, 9, and 10), and the superior frontal gyri (BA 8 and 9). The second significant cluster had a peak activation at $X Y Z=-32,-72,44$ (BA 7) and an extent of 118 voxels. The activation extended to the left precuneus (BA 7 and 19), left superior (BA 7), and inferior parietal lobules (BA 7, 19, and 39). Table 2 lists the cluster sizes, the number of voxels from each cluster

Table 3

Significant clusters obtained from analyzing the contrast between correct case judgements on bivalent stimuli in Block 2 vs. correct case judgements on univalent stimuli in the same position averaged over Block 1 and Block 3 (i.e., the "Stroop" effect)

\begin{tabular}{|c|c|c|c|c|c|c|c|}
\hline \multicolumn{3}{|l|}{ Clusters } & \multicolumn{5}{|l|}{ Peak } \\
\hline Cortical regions & Size & Overlapping BAs & $X$ & $Y$ & $Z$ & $t$ & BA \\
\hline Cluster 1 & 3525 & $6,8,9,10,24,32,33,42$ & -2 & 30 & 46 & 9.20 & 8 \\
\hline Superior frontal gyrus & 878 & $6,8,9,10$ & & & & & \\
\hline Middle frontal gyrus & 28 & 6 & & & & & \\
\hline Medial frontal gyrus & 1576 & $6,8,9,10,32$ & & & & & \\
\hline Cluster 2 & 2997 & $6,8,9,13,25,38,44,45,46,47$ & -46 & 8 & 22 & 7.54 & 9 \\
\hline Superior frontal gyrus & 39 & $6,8,9$ & & & & & \\
\hline Middle frontal gyrus & 698 & $6,8,9,46$ & & & & & \\
\hline Inferior frontal gyrus & 716 & $6,9,13,44,45,46,47$ & & & & & \\
\hline Superior temporal gyrus & 15 & 38 & & & & & \\
\hline Anterior cingulum & 17 & 25 & & & & & \\
\hline Precentral gyrus & 139 & $6,9,44$ & & & & & \\
\hline Lentiform gyrus & 91 & None & & & & & \\
\hline Caudate & 439 & None & & & & & \\
\hline Sub-gyral & 223 & None & & & & & \\
\hline Extra-nuclear & 513 & 13,47 & & & & & \\
\hline Cluster 3 & 2401 & $6,8,9,10,11,13,38,44,45,46,47$ & 42 & 38 & 22 & 6.59 & 46 \\
\hline Superior temporal gyrus & 138 & 38 & & & & & \\
\hline Middle frontal gyrus & 1009 & $6,8,9,10,11,45,46,47$ & & & & & \\
\hline Inferior frontal gyrus & 1030 & $9,10,44,45,46,47$ & & & & & \\
\hline Precentral gyrus & 18 & $6,9,44$ & & & & & \\
\hline Sub-gyral & 105 & 10,46 & & & & & \\
\hline Extra-nuclear & 49 & 13,47 & & & & & \\
\hline Cluster 4 & 797 & $2,7,19,22,39,40$ & -58 & -36 & 48 & 7.48 & 40 \\
\hline Supramarginal gyrus & 32 & 40 & & & & & \\
\hline Angular gyrus & 23 & 39 & & & & & \\
\hline Precuneus & 91 & 7,19 & & & & & \\
\hline Postcentral gyrus & 14 & 2,40 & & & & & \\
\hline Sub-gyral & 17 & 39 & & & & & \\
\hline Cluster 5 & 408 & $10,11,47$ & -42 & 48 & -8 & 7.45 & 11 \\
\hline Middle frontal gyrus & 330 & $10,11,47$ & & & & & \\
\hline Inferior frontal gyrus & 41 & 10,47 & & & & & \\
\hline Cluster 6 & 138 & None & 0 & -16 & -22 & 5.54 & None \\
\hline Pons & 30 & None & & & & & \\
\hline Midbrain & 107 & None & & & & & \\
\hline
\end{tabular}

Note. Activations are listed by cluster (left column) and peak activations (right column). A random effects analysis with cutoffs of $p<0.001$ uncorrected at the voxel level, and $p<.05$ corrected at the cluster level was used. Talairach region voxel counts may not equal cluster total voxel counts due to interhemispheric or non-cortical locations. BA=Brodmann's Area. $t=t$ value for peak activation. All clusters $\leq 10$ voxels in size have been omitted from the table. Sub-gyral includes Fornix, Putamen, Caudate, Nucleus Accumbens, Lateral and Medial Globus Palidus, Anterior Commissure, and Corpus Callosum. 
falling in the anatomical locations mentioned above, and peak cluster $t$ values.

Since the behavioral results yielded a significant interaction between the bivalency effect and task, we carried out a parallel analysis for the fMRI results. This analysis involved analyzing the contrast image for the quadratic effect at the random effects level as a function of Task, and masking inclusively with the "bivalency effect" image displayed in Table 2 and Fig. 2. No significant clusters were found, suggesting that the clusters of activation presented in Fig. 2 do not depend on the task being carried out.

We also computed the activation associated with the "Stroop" effect, using the parallel analysis that was described above for the behavioral data. As was the case for the bivalency effect, the largest cluster was dominated by dACC and medial prefrontal activations, and a significant cluster in the left parietal cortex was also observed, as well as 4 other clusters centered in the left and right dorsolateral prefrontal cortex, inferior frontal gyrus, and midbrain (see Table 3). The dACC activations appeared stronger for the "Stroop" effect compared to the bivalency effect. For example, the $t$ value for the dACC cluster peak $(X Y Z=-2,30,46)$ was 9.20 , and that for the left parietal cortex $(X Y Z=-58,-36,48)$ was 7.48, for the "Stroop" effect were larger than the to $t(28)=6.01$ $(X Y Z=4,40,34)$, and $t(28)=4.88(X Y Z=-32,-72,44)$, respectively, for the bivalency effect. However, a direct statistical comparison of the "Stroop" and bivalency effects at the random effect level using an uncorrected $p$-value of 0.001 at the voxel level, and .05 corrected at the cluster level, resulted in no significant differences.

\section{Discussion}

When switching tasks, if stimuli are presented that cue two of the tasks in the task set (i.e., bivalent stimuli), performance slowing is observed on all tasks, including those not cued by the bivalent stimulus. This has been coined the bivalency effect, and may reflect adaptive tuning of the response style under conditions that appear to require adjustments in control over the course of action. Recent work on the function of the dorsal anterior cingulate (dACC) cortex has suggested that this neural region may be recruited under such conditions. We used tightly matched experimental and control conditions to isolate the bivalency effect, and as predicted, dACC activation was observed, supporting an account stating that the role of the dACC is to signal a break in task inertia in order to adaptively tune the response style due to conditions that may require adjustments in control over the course of action.

This method of investigating task switching diverges from that typically reported in the literature. The majority of task switching studies report comparisons between task switching and task repetition, and dACC activation is consistently observed for that comparison (e.g., Slagter et al., 2006; Yeung et al., 2006). In addition, as was reported in Table 3, dACC activation is commonly observed in investigations of "Stroop-like" interference, whereby responses to incongruent (i.e., bivalent) stimuli are compared to those from neutral (i.e., univalent) or congruent stimuli (Kerns et al., 2004; Liu et al., 2004; Milham et al., 2001; van Veen et al., 2001), and this comparison has also been made within the context of task switching (Crone et al., 2006; Ruff et al., 2001; Woodward et al., 2006b). In the current study, neither the switch vs. repeat nor the incongruent vs. neutral comparisons were of primary interest; instead, comparisons were made between different task switching conditions in response to neutral (i.e., univalent) stimuli only.
Despite switching between identical tasks on identical stimuli, dACC activation was still observed after a break in inertia, and adaptive adjustment of the response style was invoked by presentation of bivalent stimuli. The observed "Stroop-like" activation did not differ significantly from the bivalency effect activation, suggesting that the dACC activation associated with the Stroop effect may be partly due to breaking inertia and adaptive adjustment of the response style.

These data clearly fit well with the "breaking inertia" (Paus, 2001) account of dACC activation, and with accounts suggesting that the role of the dACC is to detect volatility (non-stationarity) in the environment, such that activation increases when the current environment is changing in a way that may require further adjustment of responses (Behrens et al., 2007). It must also be considered whether the current data fit with the interpretation that dACC activation is attributable to conflict detection (Botvinick et al., 1999; Carter et al., 2000; Cohen et al., 2000; MacDonald et al., 2000). The conflict detection account attributes dACC activity to simultaneous activation of incompatible response channels (Botvinick et al., 2001). Extension of the conflict account to the current data is somewhat problematic, because, unlike in past studies, in the current study the "Stroop-like" conflict (incongruent vs. neutral, or bivalent vs. univalent) was equated across the experimental and control conditions, and all RT slowing and dACC activation was observed on univalent trials, caused by previously encountered bivalent stimuli. Some fMRI data are available suggesting that past conflict can affect the current trial and elicits dACC activation (Kerns et al., 2004; Woodward et al., 2006b), but these studies focused on incongruent/bivalent trials compared to a control condition.

In order to apply the conflict detection account to the current data, it must be presumed that stimulus- and/or response-based conflict from past trials is present even when responding to univalent stimuli, some of which do not share any properties with the bivalent stimulus (i.e., Stroop-like conflict is not required on the current trial to activate the dACC). That dACC activation could be expected even in the absence of Stroop-like conflict has been supported by a mathematical model of a stem completion task (Botvinick et al., 2001), suggesting that a period of subthreshold, simultaneous activation of competing response/stimulus processing channels may manifest as ACC activity even when conflict is not elicited by the current stimulus. Also, in the same paper (simulation 2), the authors illustrate how conflict on any trial can affect response priming across trials, which would also account for the bivalency effect. In order to extend this conflict detection account of dACC activation to the bivalency effect, more data will be required showing that conflict between Task $\mathrm{A}$ and $\mathrm{B}$ can also be present when carrying out Task $\mathrm{C}$ under task switching conditions. Relatedly, there is also evidence that even without increased conflict or errors, basic detection of a higher likelihood of errors can lead to dACC activation (Brown and Braver, 2005). Although the frequency of errors was very low in all blocks, this interpretation may fit with the current data because there were slightly more errors in Block 2 than in the average of Blocks 1 and 3 , even on univalent trials. Another possibility is that detection of a higher likelihood of errors is simply a special case of detection of conditions that may require adjustments in control over the course of action.

The dACC activation associated with the bivalency effect was part of a cluster that included activations not only in the dACC, but also the medial and superior frontal cortex, primarily overlapping 
with Broadmann's Areas 6,8, and 9. Medial activations extending superiorly to the dACC into the pre-supplementary motor regions (pre-SMA) are commonly reported in AACC studies (Luks et al., 2002; Ruff et al., 2001; van Veen et al., 2001). Studies testing different types of conflict have concluded that semantic conflict activates more dorsal regions of the $\mathrm{AACC}$, extending into medial frontal regions (van Veen and Carter, 2005). Other studies have suggested that the pre-SMA regions (e.g., BA 8 and 6) are involved in "decisions under uncertainty" (Volz et al., 2003, 2004), free selection of responses (Lau et al., 2004), and response conflict monitoring (Garavan et al., 2003). This suggests that the dACC and the pre-SMA may both be involved in the bivalency effect, the latter contributing to response selection under conditions of instability, combined with the dACC signalling that a change in response style is required.

The parietal activation observed for both the bivalency and "Stroop" effect is often attributed to attention shifting (Liu et al., 2003; Slagter et al., 2006), but is also involved in working memory, proposed to be involved in storing phonological material (Baddeley, 2003; Cairo et al., 2004; Smith and Jonides, 1998; Woodward et al., 2006a). Both of these interpretations tie in with the results reported here, as detection that a change in responding is needed may invoke a shift in attention from the current task rhythm to a new one, and a verbal representation may be invoked in order to carry out this adjustment. Alternatively, task instructions may be called into working memory in order to guide performance under conditions of perceived ambiguity, and participants may begin to track which task they just executed in order to determine the current task. Supporting this interpretation is the finding that articulatory suppression, possibly affecting the parietal activation observed in the current study, slows task switching performance (Emerson and Miyake, 2003; Miyake et al., 2004).

The bivalency cost when switching tasks is a generalized RT slowing that occurs whenever bivalent stimuli are encountered, and is characterized by slower responding due to the break in inertia required to carry out adaptive tuning of the response style. The dACC activation that has been associated with the bivalency effect suggests that one of its roles in task switching is to monitor internal states for conditions that may require adjustments in control over the course of action, and to signal a break in task inertia in order to adaptively tune the response style to adapt to these new conditions. This result may also support an extension of the conflict monitoring account of dACC activation to situations where conflict occurred on past trials (i.e., conflict is not elicited by the current stimulus).

\section{Acknowledgments}

This research was supported by a Discovery Grant from the Natural Sciences and Engineering Research Council (NSERC) to TSW and a Michael Smith Foundation for Health Research Scholar Award to TSW. The authors would like to thank MR technicians Trudy Shaw, Paula Coutts, Sylvia Renneberg, and Jennifer McCord for assistance with data collection, and Dr. Sara Weinstein and Dr. Judith Shedden for comments on early versions of this manuscript.

\section{References}

Annett, M., 1970. A classification of hand preference by association analysis. British Journal of Psychology 303-321.
Baddeley, A., 2003. Working memory: looking back and looking forward. Nature Reviews. Neuroscience 4, 829-839.

Behrens, T.E., Woolrich, M.W., Walton, M.E., Rushworth, M.F., 2007. Learning the value of information in an uncertain world. Nature Neuroscience 10, 1214-1221.

Botvinick, M., Nystrom, L.E., Fissell, K., Carter, C.S., Cohen, J.D., 1999. Conflict monitoring versus selection-for-action in anterior cingulate cortex. Nature 402, 179-181.

Botvinick, M.M., Braver, T.S., Barch, D.M., Carter, C.S., Cohen, J.D., 2001. Conflict monitoring and cognitive control. Psychological Review 108, 624-652.

Brown, J.W., Braver, T.S., 2005. Learned predictions of error likelihood in the anterior cingulate cortex. Science 307, 1118-1121.

Cairo, T.A., Liddle, P.F., Woodward, T.S., Ngan, E.T.C., 2004. The influence of working memory load on phase specific patterns of cortical activity. Cognitive Brain Research 21, 377-387.

Carter, C.S., Macdonald, A.M., Botvinick, M., Ross, L.L., Stenger, V.A., Noll, D., Cohen, J.D., 2000. Parsing executive processes: strategic vs. evaluative functions of the anterior cingulate cortex. Proceedings of the National Academy of Sciences of the United States of America 97, 1944-1948.

Cohen, J.D., Botvinick, M., Carter, C.S., 2000. Anterior cingulate and prefrontal cortex: who's in control? Nature Neuroscience 3, 421-423.

Crone, E.A., Wendelken, C., Donohue, S.E., Bunge, S.A., 2006. Neural evidence for dissociable components of task-switching. Cerebral Cortex $16,475-486$.

Emerson, M.J., Miyake, A., 2003. The role of inner speech in task switching: a dual-task investigation. Journal of Memory and Language 48, 148-168.

Forstmann, B.U., Brass, M., Koch, I., von Cramon, D.Y., 2006. Voluntary selection of task sets revealed by functional magnetic resonance imaging. Journal of Cognitive Neuroscience 18, 388-398.

Garavan, H., Ross, T.J., Kaufman, J., Stein, E.A., 2003. A midline dissociation between error-processing and response-conflict monitoring. NeuroImage 20, 1132-1139.

Gehring, W.J., Taylor, S.F., 2004. When the going gets tough, the cingulate gets going. Nature Neuroscience 7, 1285-1287.

Hayden, B.Y., Platt, M.L., 2006. Fool me once, shame on me-fool me twice, blame the ACC. Nature Neuroscience 9, 857-859.

Holroyd, C.B., Coles, M.G., 2002. The neural basis of human error processing: reinforcement learning, dopamine, and the error-related negativity. Psychological Review 109, 679-709.

Josephs, O., Turner, R., Friston, K., 1997. Event-related fMRI. Human Brain Mapping 5, 1-7.

Kerns, J.G., Cohen, J.D., MacDonald III, A.W., Cho, R.Y., Stenger, V.A., Carter, C.S., 2004. Anterior cingulate conflict monitoring and adjustments in control. Science 303, 1023-1026.

Lau, H.C., Rogers, R.D., Ramnani, N., Passingham, R.E., 2004. Willed action and attention to the selection of action. NeuroImage 21, 1407-1415.

Liu, T., Slotnick, S.D., Serences, J.T., Yantis, S., 2003. Cortical mechanisms of feature-based attentional control. Cerebral Cortex 13, 1334-1343.

Liu, X., Banich, M.T., Jacobson, B.L., Tanabe, J.L., 2004. Common and distinct neural substrates of attentional control in an integrated Simon and spatial Stroop task as assessed by event-related fMRI. NeuroImage 22, 1097-1106

Luks, T.L., Simpson, G.V., Feiwell, R.J., Miller, W.L., 2002. Evidence for anterior cingulate cortex involvement in monitoring preparatory attentional set. NeuroImage 17, 792-802.

MacDonald, A.W., Cohen, J.D., Stenger, V.A., Carter, C., 2000. Dissociating the role of the dorsolateral prefrontal and anterior cingulate cortex in cognitive control. Science 288 (9), 1835-1838.

Milham, M.P., Banich, M.T., Webb, A., Barad, V., Cohen, N.J., Wszalek, T., Kramer, A.F., 2001. The relative involvement of anterior cingulate and prefrontal cortex in attentional control depends on nature of conflict. Cognitive Brain Research 12, 467-473.

Miyake, A., Emerson, M.J., Padilla, F., Ahn, J.C., 2004. Inner speech as a retrieval aid for task goals: the effects of cue type and articulatory suppression in the random task cuing paradigm. Acta Psychol. (Amst) $115,123-142$ 
Parris, B.A., Thai, N.J., Benattayallah, A., Summers, I.R., Hodgson, T.L., 2007. The role of the lateral prefrontal cortex and anterior cingulate in stimulus-response association reversals. Journal of Cognitive Neuroscience $19,13-24$.

Paus, T., 2001. Primate anterior cingulate cortex: where motor control, drive and cognition interface. Nature Reviews. Neuroscience 2, 417-424.

Peterson, B.S., Skudlarski, P., Gatenby, J.C., Zhang, H., Anderson, A.W., Gore, J.C., 1999. An fMRI study of Stroop word-color interference: evidence for cingulate subregions subserving multiple distributed attentional systems. Biological Psychiatry 45, 1237-1258.

Ridderinkhof, K.R., Ullsperger, M., Crone, E.A., Nieuwenhuis, S., 2004 The role of the medial frontal cortex in cognitive control. Science 306, 443-447.

Ruff, C.C., Woodward, T.S., Laurens, K.R., Liddle, P.F., 2001. The role of the anterior cingulate cortex in conflict processing: evidence from reverse Stroop interference. NeuroImage 14, 1150-1158.

Rushworth, M.F., Walton, M.E., Kennerley, S.W., Bannerman, D.M., 2004. Action sets and decisions in the medial frontal cortex. Trends in Cognitive Sciences 8, 410-417.

Slagter, H.A., Weissman, D.H., Giesbrecht, B., Kenemans, J.L., Mangun, G.R., Kok, A., Woldorff, M.G., 2006. Brain regions activated by endogenous preparatory set shifting as revealed by fMRI. Cognitive, Affective \& Behavioral Neuroscience 6, 175-189.

Smith, E.E., Jonides, J., 1998. Neuroimaging analyses of human working memory. Proceedings of the National Academy of Sciences of the United States of America 95, 12061-12068.

Stuss, D.T., 2006. Frontal lobes and attention: processes and networks, fractionation and integration. Journal of the International Neuropsychological Society $12,261-271$. van Veen, V., Carter, C.S., 2005. Separating semantic conflict and response conflict in the Stroop task: a functional MRI study. NeuroImage 27, 497-504.

van Veen, V., Cohen, J.D., Botvinick, M.M., Stenger, V.A., Carter, C.S., 2001. Anterior cingulate cortex, conflict monitoring, and levels of processing. NeuroImage 14, 1302-1308.

Volz, K.G., Schubotz, R.I., von Cramon, D.Y., 2003. Predicting events of varying probability: uncertainty investigated by fMRI. NeuroImage 19, 271-280.

Volz, K.G., Schubotz, R.I., von Cramon, D.Y., 2004. Why am I unsure? Internal and external attributions of uncertainty dissociated by fMRI. NeuroImage 21, 848-857.

Williams, Z.M., Bush, G., Rauch, S.L., Cosgrove, G.R., Eskandar, E.N., 2004. Human anterior cingulate neurons and the integration of monetary reward with motor responses. Nature Neuroscience 7, 1370-1375.

Woodward, T.S., Meier, B., Tipper, C., Graf, P., 2003. Bivalency is costly: bivalent stimuli elicit cautious responding. Experimental Psychology 50, 233-238.

Woodward, T.S., Cairo, T.A., Ruff, C.C., Takane, Y., Hunter, M.A., Ngan, E.T.C., 2006a. Functional connectivity reveals load dependent neural systems underlying encoding and maintenance in verbal working memory. Neuroscience 139, 317-325.

Woodward, T.S., Ruff, C.C., Ngan, E.T., 2006b. Short- and long-term changes in anterior cingulate activation during resolution of task-set competition. Brain Research 1068, 161-169.

Woodward, T.S., Meier, B., Metzak, P.D., Graf, P., submitted for publication. The bivalency effect in task switching.

Yeung, N., Nystrom, L.E., Aronson, J.A., Cohen, J.D., 2006. Between-task competition and cognitive control in task switching. The Journal of Neuroscience 26, 1429-1438. 